\title{
Impaired intestinal barrier function in a mouse model of hyperuricemia
}

\author{
YINGJIE GUO ${ }^{1}$, HAILONG LI ${ }^{2}$, ZHEN LIU ${ }^{2}$, CHANGGUI LI ${ }^{2}$, YUNQING CHEN ${ }^{3}$, \\ CHEN JIANG $^{1}$, YANAN YU ${ }^{1}$ and ZIBIN TIAN ${ }^{1}$ \\ ${ }^{1}$ Department of Gastroenterology, The Affiliated Hospital of Qingdao University; \\ ${ }^{2}$ Institute of Metabolic Diseases, Qingdao University; ${ }^{3}$ Department of Pathology, \\ The Affiliated Hospital of Qingdao University, Qingdao, Shandong 266000, P.R. China
}

Received March 13, 2019; Accepted July 30, 2019

DOI: $10.3892 / \mathrm{mmr} .2019 .10586$

\begin{abstract}
Previous studies have demonstrated the effects of hyperuricemia on the damage to target organs, including the kidneys, joints and the heart. However, it is unclear whether hyperuricemia results in damage to the intestines. The aim of the present study was to investigate intestinal barrier dysfunction in a mouse model of hyperuricemia constructed by knocking out the urate oxidase (Uox) gene. The morphology of the intestine was assessed via hematoxylin and eosin, and alcian blue staining. The serum and intestinal tissue levels of uric acid, tumor necrosis factor (TNF)- $\alpha$ and interleukin (IL)-6, in addition to the presence of uremic toxins in the serum, were assessed. The levels of diamine oxidase (DAO), D-lactate (D-LAC) and endotoxins in the serum, which are markers of the intestinal permeability, were measured using ELISA. The expression of the intestinal tight junction proteins zona occludens-1 (ZO-1) and occludin were detected by reverse transcription-quantitative polymerase chain reaction, western blotting and immunohistochemical analysis. The Uox-knockout mice spontaneously developed hyperuricemia. Histopathological analysis indicated notable intestinal defects including sparse villi, mucosal edema and a declining mucus layer in hyperuricemic mice. The expression levels of ZO-1 and occludin in the intestines were downregulated, and the serum levels of DAO, D-LAC and endotoxins were higher in the hyperuricemic mice, compared with control mice. The serum and intestinal tissue levels of IL- 6 and TNF- $\alpha$ were significantly increased. Additionally, the expression levels of the serum uremic toxins, serum creatinine, blood urea nitrogen were significantly increased in hyperuricemic mice compared
\end{abstract}

Correspondence to: Professor Zibin Tian or Dr Yanan Yu, Department of Gastroenterology, The Affiliated Hospital of Qingdao University, 16 Jiangsu Road, Qingdao, Shandong 266000, P.R. China E-mail: tianzb@qduhospital.cn

E-mail: yananyu1985@163.com

Key words: hyperuricemia, urate oxidase, intestine barrier, tight junction, inflammation with the control mice, while only a marked increase in indoxyl sulfate (IS) and p-cresol sulfate was reported. Collectively, the results of the present study suggested that intestinal barrier dysfunction and subsequent enhanced intestinal permeability may occur as a result of hyperuricemia in mice. Furthermore, we proposed that the loss of intestinal epithelium barrier function may be associated with uric acid-induced inflammatory responses; however, further investigation is required.

\section{Introduction}

Hyperuricemia is a common metabolic disease caused by a disorder of purine metabolism in humans (1). In the majority of mammals, uric acid (UA) is converted to the more soluble form allantoin by urate oxidase (Uox), which is removed from the body. However, the Uox gene has undergone mutational silencing during the evolution of humans and hominoid apes; thus, humans are vulnerable to hyperuricemia (2). Hyperuricemia has been a global public health concern with an increasing incidence and prevalence in recent years $(3,4)$. Hyperuricemia is a major risk factor for gout (5). In total, 10-20\% patients with hyperuricemia may progress to gout (5). In addition, studies have suggested that hyperuricemia may also contribute to the development and pathogenesis of metabolic and systemic pathologic diseases, including diabetes, metabolic syndrome, hypertension, atherosclerosis and chronic kidney disease $(6,7)$.

The intestine is the primary organ responsible for the clearance of UA and one third of UA in the blood is removed through the intestines $(8,9)$. Certain patients with hyperuricemia present with gastrointestinal symptoms; however, the precise effects of hyperuricemia on the intestines remain unclear. A previous study showed that hyperuricemia is associated with an increased risk of colorectal carcinoma (10). Additionally, it was documented that supplementing exogenous UA via an intra-intestinal injection induces the influx of heterophils into the intestines of rabbits (11). Nevertheless, supplementing exogenous UA in experimental animals within a short time does not mimic human hyperuricemia adequately as the majority of cases of hyperuricemia in humans are sustained for at least a few years. In our previous study, a mouse model of hyperuricemia was established by knocking out the Uox 
gene (12). The Uox-knockout (KO) mice spontaneously developed hyperuricemia (serum UA concentration $>7 \mathrm{mg} / \mathrm{dl}$ ) and survived $\leq 62$ weeks (12). Therefore, we employed this model to investigate the effects of hyperuricemia effects on the intestines. In the present study, intestinal barrier function and intestinal permeability was assessed in the hyperuricemia mouse model, and the serum and intestinal tissue levels of UA, tumor necrosis factor (TNF)- $\alpha$ and interleukin (IL)-6, as well as serum uremic toxins were measured. We aimed to investigate intestinal barrier dysfunction in a mouse model of hyperuricemia, and further understand the underlying mechanisms.

\section{Materials and methods}

Animal model. In our previous study, a hyperuricemia mouse model was generated by knocking out the Uox gene using the transcription activator-like effector nuclease technique (12). The male Uox-KO mice and their wild-type (WT) counterparts ( $n=8$ in each group) were used in the present study. The mice from 4 weeks (weight, 13-15 g) to 24 weeks (weight, 28-32 g) after birth were maintained individually in a temperature-controlled room at $22-24^{\circ} \mathrm{C}$ and $40-60 \%$ relative humidity under a 12-h light/dark cycle with ad libitum access to food and water. Blood and urine samples of all the animals were collected in the morning. The blood samples were centrifuged at $1,511 \mathrm{xg}$ for $15 \mathrm{~min}$ at $4^{\circ} \mathrm{C}$ to obtain the serum. The mice were euthanized at 24 weeks after birth using $\mathrm{CO}_{2}$ with $25 \%$ volume displacement per minute, and mortality was confirmed by observing respiration and by using the corneal reflection method. The intestines were harvested, and a section of the intestinal tissues were cut and washed with cold normal saline to remove blood; after drying on filter papers at room temperature, the intestinal samples were weighed, then immediately homogenized on ice. The homogenates were centrifuged at 5,000 x g for $10 \mathrm{~min}$ at $4^{\circ} \mathrm{C}$ to obtain the supernatants. All procedures were performed with the approval of The Animal Research Ethics Committee of The Affiliated Hospital of Qingdao University (approval no. AHQU20140612A).

Measurement of UA. Serum UA levels were analyzed in the mice from 4 to 24 weeks, and the levels of UA in the urine and the supernatant of the intestinal homogenate were measured at 24 weeks of age. The levels of UA in serum, urine and supernatant were measured using a UA determination kit R-i/ii (Beijing Leadman Biochemistry Co., Ltd.). The detection process was completed with an automatic biochemical analyzer (Toshiba Corporation, Minato, Japan) according to the manufacturer's protocols.

Histological examination. Intestinal samples were fixed by immersion in $10 \%$ neutral formalin at room temperature for $48 \mathrm{~h}$, and subsequently embedded in paraffin. The paraffin block of tissue was sliced into $4-\mu \mathrm{m}$-thick sections, and stained with hematoxylin and eosin for $5 \mathrm{~min}$ at room temperature. Alcian blue staining was performed as described previously; the sections were stained with Alcian blue for $30 \mathrm{~min}$ at room temperature (13). Pathological changes in the intestine were observed under a light microscope (magnification, $\mathrm{x} 400$;
Axiovert 200; Zeiss AG) and evaluated by an experienced pathologist.

Reverse transcription-quantitative polymerase chain reaction $(R T-q P C R)$. Total RNA was extracted from intestinal tissue using RNAiso Plus (Takara Bio, Inc.) and was reverse-transcribed into cDNA using a PrimeScript RT reagent kit with gDNA Eraser (Takara Bio, Inc.), according to the manufacturer's protocols. qPCR reaction was performed in a $25 \mu \mathrm{l}$ reaction volume, using TB Green Premix Ex Taq reagent (Takara Bio, Inc.) using a fluorescent PCR device (CFX96, Bio-Rad Laboratories, Inc.). The thermocycling conditions were: i) $95^{\circ} \mathrm{C}$ For $30 \mathrm{sec}$; and ii) 40 cycles of $95^{\circ} \mathrm{C}$ for $5 \mathrm{sec}$ and $60^{\circ} \mathrm{C}$ for $30 \mathrm{sec}$. The primer sequences were as follows: Zona occludens-1 (ZO-1) forward, 5'CCACCTCTG TCCAGCTCTTC-3', reverse, 5'CACCGGAGTGATGGT TTTCT-3; Occludin forward, 5'CCTCCAATGGCAAAG TGAAT-3', reverse, 5'CTCCCCACCTGTCGTGTAGT-3, and $\beta$-actin forward, 5'CTCCCTGGAGAAGAGCTATGA-3 and reverse, 5'GGCATAGAGGTCTTTACGGATG-3'. All samples were run in triplicate in a single 96 -well reaction plate. The relative expression levels were analyzed using the $2^{-\Delta \Delta \mathrm{Cq}}$ method $(14,15)$.

Immunohistochemistry detection of tight junction proteins. Immunohistochemistry for ZO-1 and occludin in intestinal tissue was performed as described previously (16). Briefly, the intestinal tissues from the Uox-KO mice and the WT mice were fixed and embedded in paraffin as aforementioned, and 4- $\mu \mathrm{m}$ sections were cut from the paraffin blocks. The sections were dewaxed in xylene (Beijing Zhongshan Golden Bridge Biotechnology Co., Ltd.; OriGene Technologies, Inc.) and antigen retrieval was performed using $10 \mathrm{mmol} / \mathrm{l}$ citrate buffer ( $\mathrm{pH}$ 6.0; Beijing Zhongshan Golden Bridge Biotechnology Co., Ltd.; OriGene Technologies, Inc.) at $120^{\circ} \mathrm{C}$ for $3 \mathrm{~min}$. Endogenous peroxidases activity were inhibited with $3 \%$ hydrogen peroxide for $10 \mathrm{~min}$ at room temperature. Then, the sections were incubated at $4^{\circ} \mathrm{C}$ overnight using rabbit anti-ZO-1 (1:400; cat. no. bs-1329R; BIOSS Biotech Co., Ltd) and rabbit anti-occludin (1:300; cat. no. A2601; ABclonal Biotech Co., Ltd.). As a negative control, sections were incubated with PBS alone. Subsequently, the sections were incubated with a goat-anti-rabbit immunoglobulin G secondary antibody (1:500; cat. no. PV9001; Beijing Zhongshan Golden Bridge Biotechnology Co., Ltd.; OriGene Technologies, Inc.) at room temperature for $1 \mathrm{~h}$ and stained with diaminobenzidine (OriGene Technologies, Inc.) at room temperature for $5 \mathrm{~min}$. The sections were counterstained with hematoxylin at room temperature for $5 \mathrm{~min}$ and analysis was conducted with an optical microscope (magnification, $x 400$ ). The immunoreactive positive signals appeared brown.

Western blotting analysis of tight junction proteins. Western blotting was performed as previously described (17). Briefly, total proteins were extracted from intestinal tissues using a protein lysis buffer $(50 \mathrm{mM}$ Tris- $\mathrm{HCl}, 150 \mathrm{mM} \mathrm{NaCl}, 1 \%$ Triton $\mathrm{X}-100,0.5 \%$ sodium deoxycholate, $0.1 \%$ SDS; Applygene) and the protein concentration was measured using a bicinchoninic acid assay kit (Pierce; Thermo Fisher Scientific, Inc.). A total of $30 \mu \mathrm{g}$ of protein from each sample were resolved using a $10 \%$ SDS-PAGE gel and transferred to a polyvinylidene difluoride 
membrane (EMD Millipore), and blocked using 5\% nonfat milk at room temperature for $1 \mathrm{~h}$. Membranes were incubated with primary antibodies at $4^{\circ} \mathrm{C}$ overnight: Rabbit anti-ZO-1 (1:1,000; cat. no. 5406, Cell Signaling Technology, Inc.), rabbit anti-occludin (1:1,000; cat. no. A2601; ABclonal Biotech Co., Ltd.) and anti-GAPDH (1:1,000; cat. no. 8884; Cell Signaling Technology,Inc.). Subsequently, the membranes were incubated at $4^{\circ} \mathrm{C}$ with a horseradish peroxidase-conjugated secondary antibody (1:10,000; cat. no. 7074; Cell Signaling Technology, Inc.) for $1 \mathrm{~h}$. An FR-980 Biological Image Analysis System (Furi) was used to detect immunoreactive bands and densitometry analysis was performed using ImageJ (version 1.8.0; National Institutes of Health).

Quantification of intestinal permeability. Diamine oxidase (DAO), D-lactate (D-LAC), and endotoxin levels in the serum are commonly regarded as indirect indicators of intestinal permeability (18). The concentrations of serum DAO, D-LAC and endotoxin were determined using ELISA kits (DAO ELISA Kit, cat. no. CSB-E10090m, Cusabio Technology LLC; D-lactate ELISA Kit, cat. no. JL48176, Shanghai Future Industrial Limited By Share Ltd.; endotoxin ELISA Kit, cat. no. CSB-E13066m, Cusabio Technology LLC), according to the manufacturer's protocols.

Analysis of serum uremic toxins. The levels of serum creatinine (SCR) and blood urea nitrogen (BUN) in serum samples $(200 \mathrm{ul})$ were measured using an automatic biochemical analyzer (Toshiba Corporation, Minato, Japan) to assess kidney function. The detection of indoxyl sulfate (IS) and p-cresol sulfate (PCS) levels in the serum were performed using a mouse indoxyl sulfate ELISA Kit and p-cresol sulfate ELISA Kit (cat. no. JL44175 and cat. no. JL48074, Shanghai Future Industrial Limited By Share Ltd.) according to the manufacturer's protocols.

ELISA measurement of cytokines in the serum and intestinal tissues. The concentrations of TNF- $\alpha$ and IL- 6 in the serum and intestinal tissues were quantified using the mouse TNF- $\alpha$ ELISA kit and mouse IL-6 ELISA kit, respectively (cat. nos. RK00027 and RK00008, ABclonal Biotech Co., Ltd.) according to the manufacturer's protocols.

Statistical analysis. All data were statistically analyzed using SPSS 21 (IBM Corp.) and GraphPad Prism 6 (GraphPad Software, Inc.). All values were presented as the mean \pm standard error of the mean. Experiments were performed in triplicate. Quantitative data was assessed using independent-sample t-tests or one-way analysis of variance followed by a Tukey's post-hoc test. $\mathrm{P}<0.05$ was considered to indicate a statistically significant difference.

\section{Results}

UA levels are elevated in the serum, urine and intestinal tissues of the Uox-KO mice. The Uox-KO mice had significantly elevated serum UA levels between $8.89-10.15 \mathrm{mg} / \mathrm{dl}$; however, the levels appeared to be stable and 3-4 fold higher compared with WT mice (Fig. 1A and B). The urinary UA concentration in Uox-KO mice was $\sim 80 \mathrm{mg} / \mathrm{dl}, \sim 10$ fold higher compared with the WT littermate controls (Fig. 1C). The UA concentration in the intestinal tissues was $\sim 1,310 \mu \mathrm{g} / \mathrm{g}$ in the hyperuricemic mice, which was significantly increased than that of the control group (Fig. 1D).

Histological observation of intestinal damage in the Uox-KO mice. Using light microscopy, regional epithelial shedding, fewer and fractured villi in the intestine of the Uox-KO mice were observed. Furthermore, mucosal and submucosal edema were observed in the Uox-KO mice. Conversely, the intestinal epithelial structure was intact, and no notable pathological changes were detected in the controls (Fig. 2A). Additionally, Uox-KO mice exhibited a notable reduction in mucins when compared with the WT mice as determined using alcian blue staining (Fig. 2B). These histological results suggested that the structure of the intestinal epithelium was disrupted in Uox-KO mice.

Expression of tight junction proteins is downregulated in Uox-KO mice. ZO-1 and occludin are considered to be the most important tight junction proteins, and contribute to the intestinal mucosal barrier function (19). Therefore, the mRNA and protein expression levels of $\mathrm{ZO}-1$ and occluding were measured in the intestinal tissues. The results of RT-qPCR revealed that the mRNA expression levels of ZO-1 and occluding were significantly downregulated in the Uox-KO mice compared with the control (Fig. 3A). Consistent with the mRNA expression results, the protein expression levels of ZO-1 and occluding in the intestinal tissues were also downregulated in the Uox-KO mice compared with the control as determined by western blotting (Fig. 3C). Immunohistochemistry demonstrated that the degree of positive staining for ZO-1 and occludin observed in the Uox-KO mice was markedly reduced compared with the control (Fig. 3B).

Intestinal permeability is increased in the Uox-KO mice. Impairments in the intestinal barrier were determined by intestinal permeability. Serum DAO, D-lactate and endotoxin levels were measured as markers of intestinal permeability. The results revealed that the serum levels of DAO, D-LAC and endotoxin were significantly increased in the Uox-KO mice compared with the WT mice ( $\mathrm{P}<0.05$; Fig. 4).

Levels of uremic toxin and cytokines are increased in the Uox-KO mice. To determine the potential underlying mechanism of intestinal barrier dysfunction in hyperuricemia, the serum uremic levels and cytokine levels were measured in the Uox-KO and WT mice. As presented in Fig. 5, the serum uremic toxins levels of SCR and BUN were significantly increased in Uox-KO mice compared with the control, whereas the levels of IS and PCS exhibited marked increases in the Uox-KO mice (Fig. 5). Thus, it is unlikely that the extent of damage observed in the hyperuricemic mice resulted from the slight increases in IS and PCS.

Inflammatory cytokines are known to contribute to intestinal damage (20). The levels of TNF- $\alpha$ and IL-6 in the serum and the intestinal homogenates were significantly higher in the Uox-KO mice compared with the WT mice (Fig. 6). The data indicated that the intestinal mucosal barrier damage in the hyperuricemic mice may be associated with the induction of the inflammatory response. 

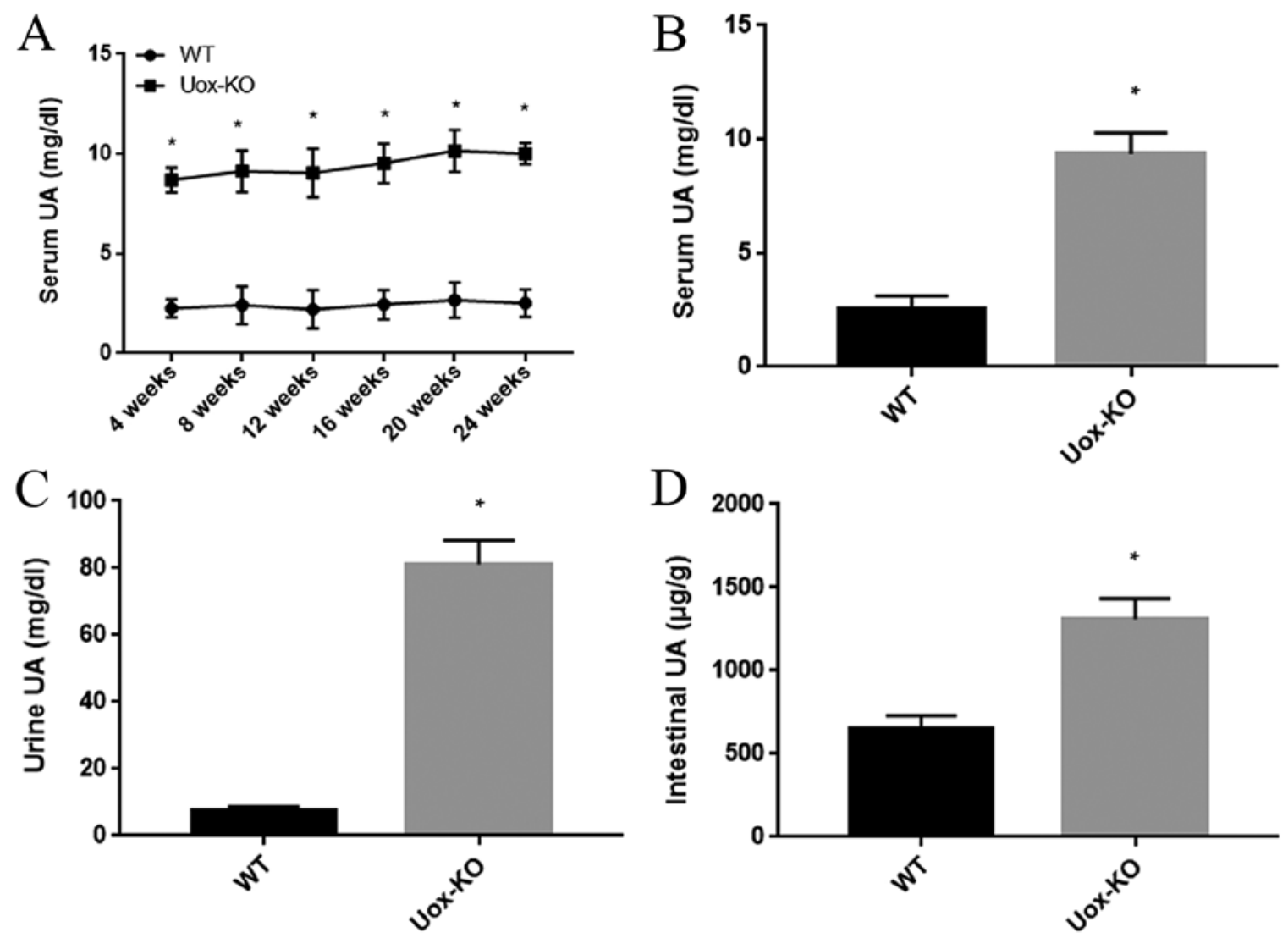

Figure 1. UA levels in serum, urine and intestinal tissues of the Uox-KO mice are increased. (A) Serum UA levels were higher in the Uox-KO mice between 4 and 24 weeks. The levels of UA in the (B) serum, (C) urine and (D) intestinal tissues of hyperuricemic mice were significantly increased compared with the WT littermate controls ${ }^{*} \mathrm{P}<0.05$ vs. WT. UA, uric acid; UOX, urate oxidase; KO, knockout; WT, wild-type.
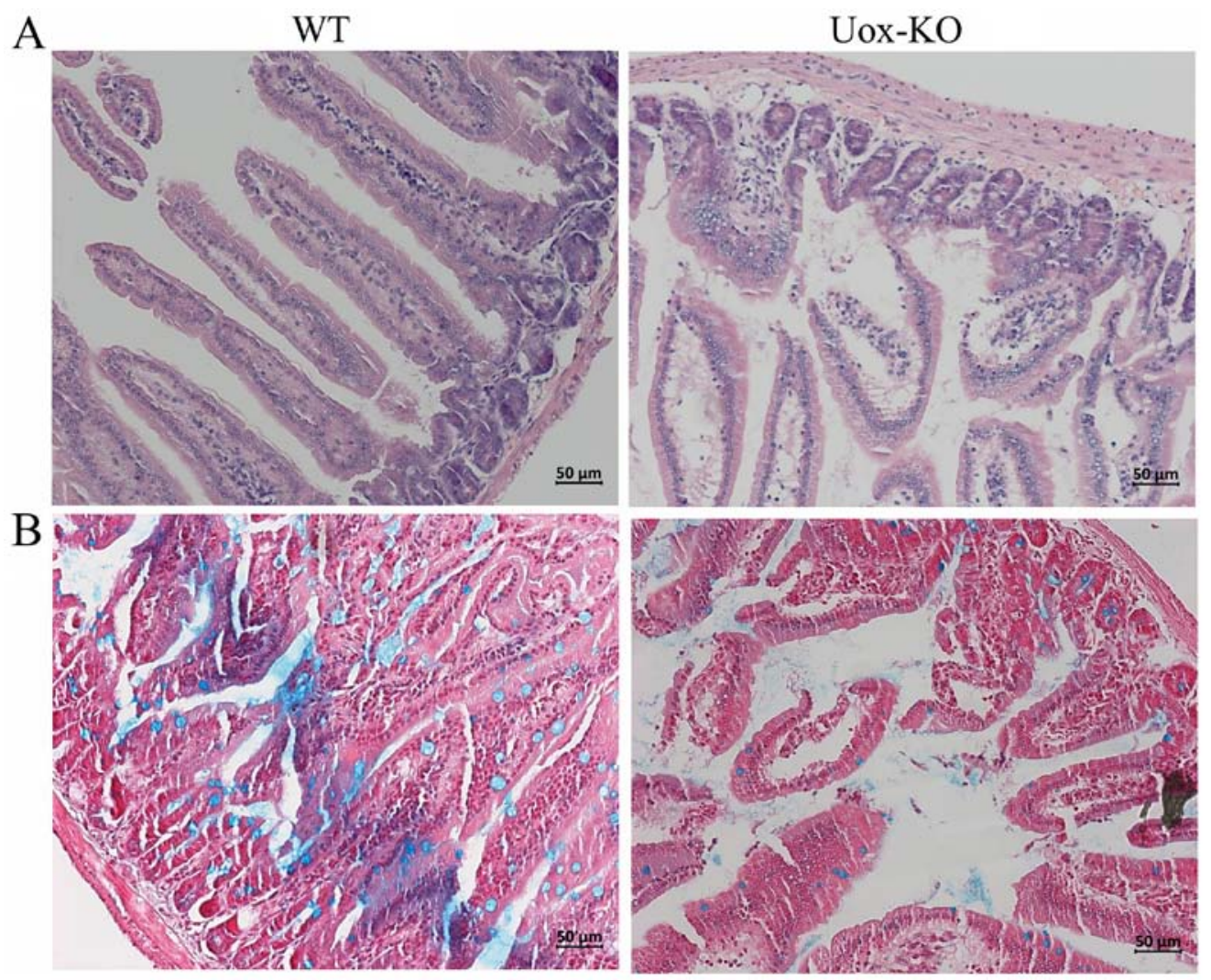

Figure 2. Histological analysis of intestinal tissue in the Uox-KO mice. (A) Hematoxylin and eosin staining showed that the intestines of hyperuricemic mice exhibited defects, including sparse intestinal villi, mucosal and submucosal edema. (B) Alcian blue staining revealed that the intestinal mucus layer was notably reduced in hyperuricemic mice compared with the control. Scale bar=50 $\mu \mathrm{m}$. UOX, urate oxidase; KO, knockout; WT, wild-type. 
A

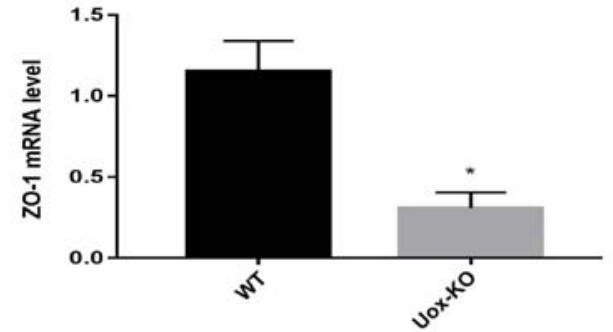

B

ZO-1
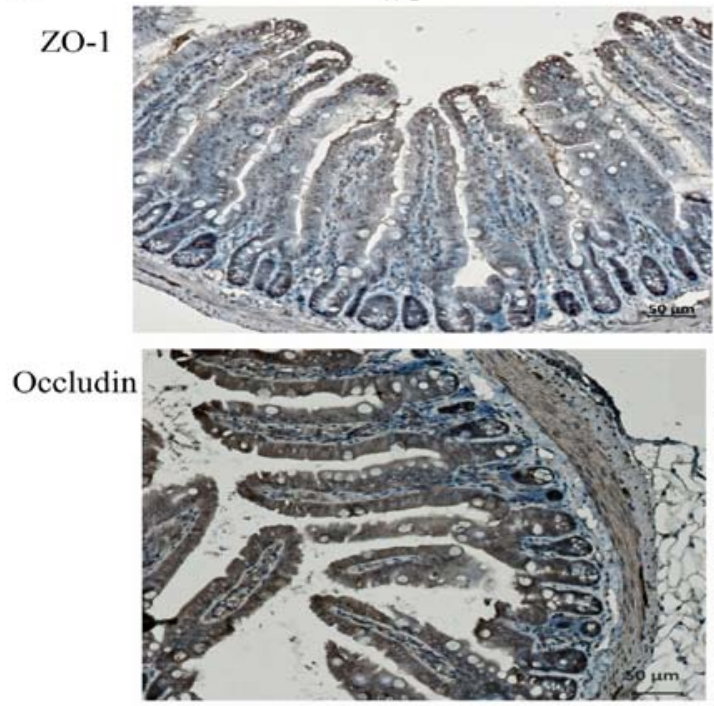

C
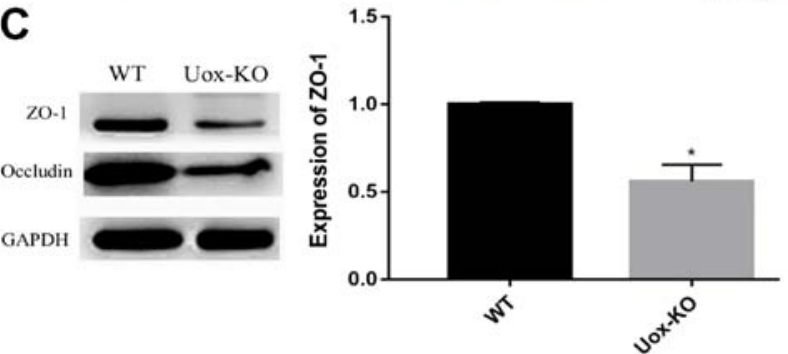

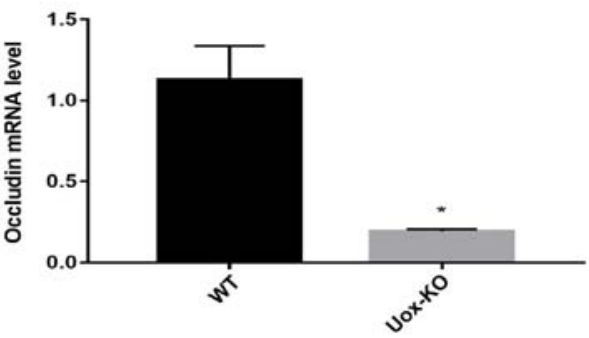

Uox-KO
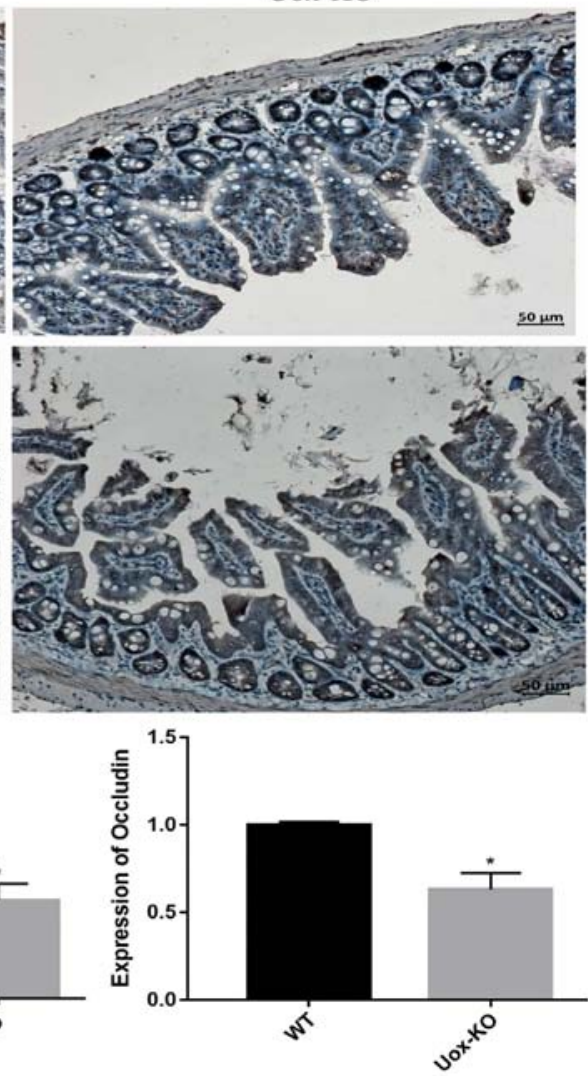

Figure 3. Expression of tight junction proteins is decreased in Uox-KO mice. (A) mRNA expression levels of ZO-1 and occluding in intestinal tissues were measured by reverse transcription-quantitative polymerase chain reaction. ${ }^{*} \mathrm{P}<0.05$ vs. control. (B) Immunohistochemical staining of $\mathrm{ZO}-1$ and occludin. Scale bar=50 $\mu \mathrm{m}$. (C) Protein expression levels of ZO-1 and occludin were analyzed by western blotting with GAPDH as a loading control and for normalization. ${ }^{*} \mathrm{P}<0.05$ vs. WT. UOX, urate oxidase; KO, knockout; WT, wild-type; ZO-1, zona occludens-1.
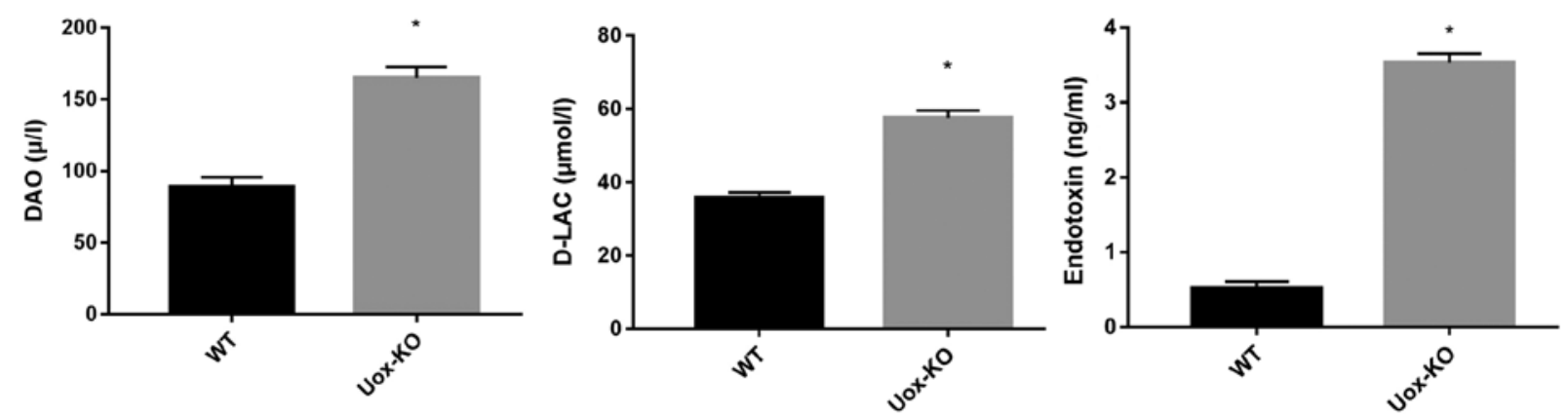

Figure 4. Serum levels of D-LAC, DAO and endotoxin are increased in Uox-KO mice. Levels of D-LAC, DAO and endotoxin were increased in the hyperuricemic group compared with the control group. "P<0.05 vs. WT. UOX, urate oxidase; KO, knockout; DAO, diamine oxidase; D-LAC, D-lactate; WT, wild-type.

\section{Discussion}

Numerous studies have confirmed that elevated serum UA levels contribute to the damage of multiple organs, including the kidneys and heart, and joints (5-7). Furthermore, a recent study indicated that UA has a role in impairment of the integrity of vascular endothelial cells (21). Notably, the structure and physiological characteristics between the vascular and 

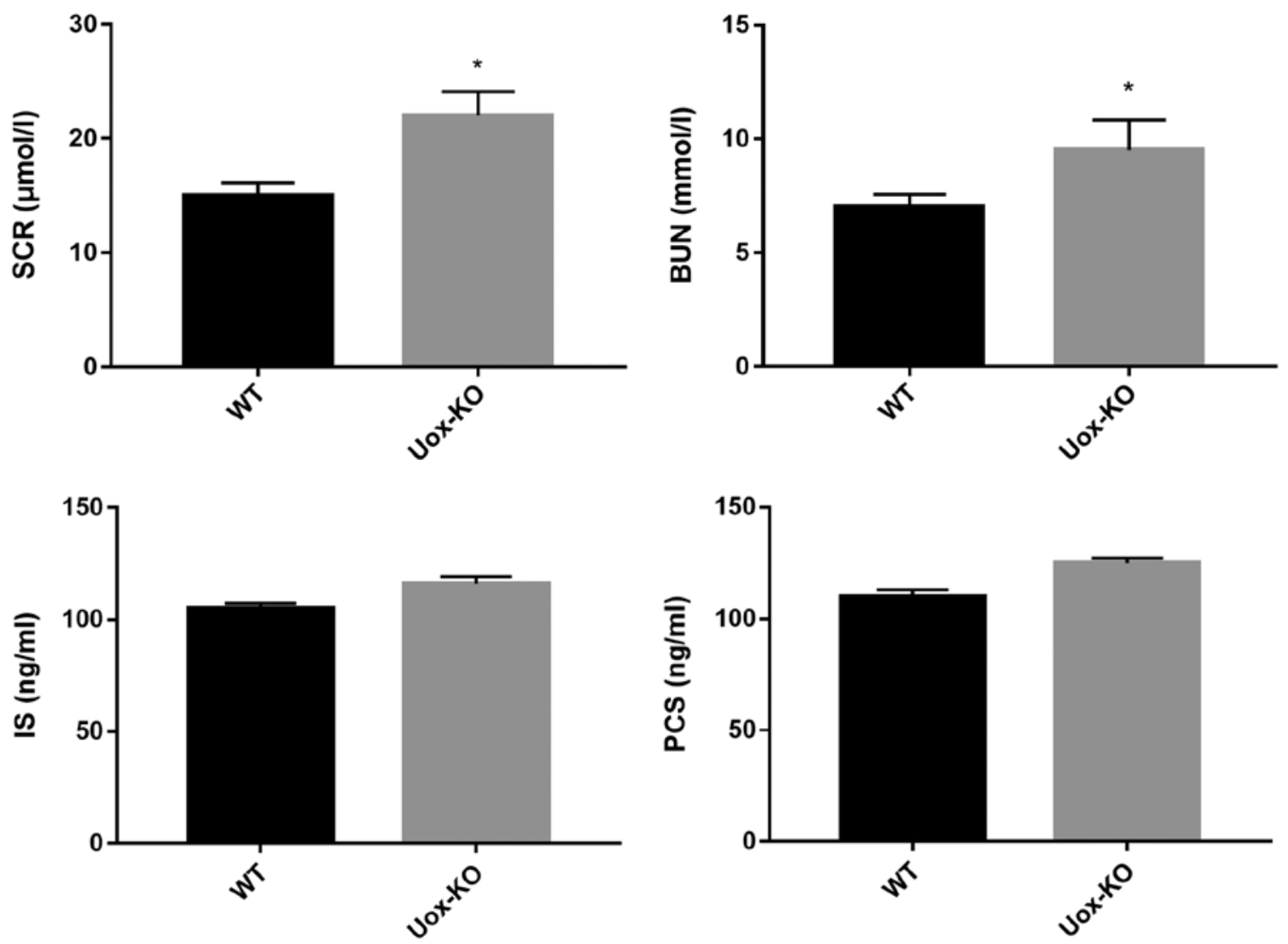

Figure 5. Uremic toxins levels are increased in the Uox-KO mice. Uremic toxins levels of SCR, BUN were significantly elevated, while those of IS and PCS were notably increased in Uox-KO mice compared with the control group. "P<0.05 vs. WT. UOX, urate oxidase; KO, knockout; SCR, serum creatinine; BUN, blood urea nitrogen; IS, indoxyl sulfate; PCS, p-cresol sulfate; WT, wild-type.
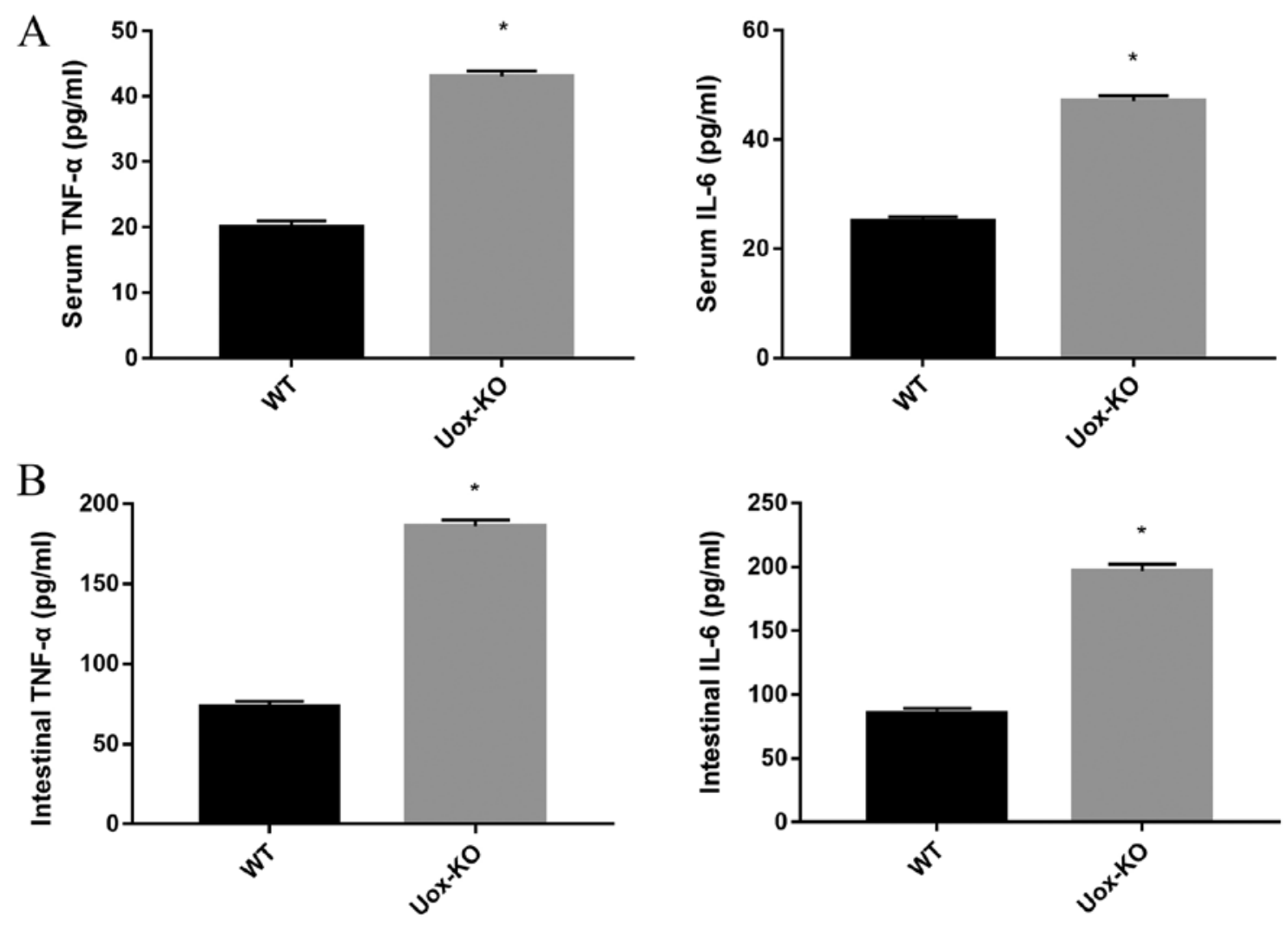

Figure 6. Cytokine levels are elevated in Uox-KO mice. The levels of TNF- $\alpha$ and IL-6 in the (A) serum (A) and (B) intestinal tissue were significantly increased in the Uox-KO mice compared with the control group. "P<0.05 vs. WT. UOX, urate oxidase; KO, knockout; TNF- $\alpha$, tumor necrosis factor- $\alpha$; IL, interleukin; WT, wild-type. 
intestinal epithelium are homologous and similar; however, few studies have addressed the possible disruptive effects of hyperuricemia on the intestine. In the present study, the Uox-KO (hyperuricemic) mice exhibited impairments in the intestinal barrier, and pathological changes in the intestines were observed, including sparse intestinal villi, mucosal and submucosal edema, suggesting that hyperuricemia may have interfered with the epithelial integrity of the intestine, contributing to intestinal damage. The findings of the present study may improve current knowledge regarding the intestinal damage caused by hyperuricemia; however, damage to other organs under these conditions requires further investigation.

Hyperuricemic mice models are generally constructed by promoting purine synthesis, inhibiting UA degradation and supplementing exogenous UA, either alone or in combination $(22,23)$. However, the UA levels in these models usually fluctuate by a large margin (23). Therefore, these models may not suited for mimicking hyperuricemia in humans. In the present study, a mouse model of hyperuricemia was established by knocking out the Uox gene. Our previous study revealed that these mice exhibited a stably increased level of UA and had developed the symptoms of compromised renal function, hypertension, aberrant glycometabolism and lipometabolism, which are similar to those observed in patients with hyperuricemia (12). In the present study, impaired intestinal barrier function was also observed in these gene deficient mice. Collectively, these data suggest that the mouse model may be a more suitable model investigating hyperuricemia and its related complications.

The importance of the intestinal epithelium for excretion has been demonstrated (24). Approximately one-third of the circulating UA is cleared via urate transporters in the intestinal enterocytes (25). The intestinal epithelium and the apical junctional complex constitute the intestinal barrier, a dynamic interface between the internal and external environments, which serves a crucial role in the maintenance of intestinal homeostasis $(26,27)$. In the present study, intestinal epithelial damage was observed in Uox-KO mice as detected by histological examination. Tight junctions between the intestinal epithelial cells are involved in the maintenance of the intestinal mucosal barrier function (28). ZO-1 and occludin are considered to be two of the primary tight junction proteins (19). In the present study, a significant decrease in the expression of ZO-1 and occluding in the intestine of $U o x-K O$ mice was reported compared with the control. The mucus layer on the epithelial surface is also an essential component of the intestinal barrier (29). The thickness of the mucus layer was also decreased in hyperuricemic mice compared with the WT mice, as determined by alcian blue staining. These results suggested that intestinal mucosal barrier function may have been damaged in the hyperuricemic mice.

Impairments in the intestinal mucosal barrier manifest as increased intestinal permeability $(30,31)$. DAO is an intracellular enzyme in the intestinal epithelium, whereas D-LAC and endotoxin are bacterial metabolites produced by the intestinal flora (32). When the permeability of the intestine is abnormally increased as a result of some sort of disruption, DAO, D-LAC and endotoxin in the lumen easily pass through the intestinal mucosa and into the peripheral blood $(32,33)$. In the present study, increases in the serum levels of DAO, D-LAC and endotoxins were observed in the hyperuricemic mice compared with the controls, suggesting enhanced intestinal permeability in the hyperuricemic mice.

The present study suggested that the intestinal barrier was damaged and intestinal permeability was increased in the hyperuricemia mouse model; however, the exact mechanism underlying injury had not been determined. Previous studies demonstrated a high concentration of uremic toxins, including IS, PCS and advanced glycation end products could result in the destruction of the intestinal barrier structure in end-stage renal disease (34-36). Additionally, chronic renal failure induced by $5 / 6$ nephrectomy results in increased levels of uremic toxins which damages the intestinal mucosal barrier in rats (37). Our previous study indicated that kidney impairment was similarly detected in the hyperuricemic Uox-KO mice, in which collapsed and necrotic nephrons and focal tubulointerstitial fibrosis were observed (12). In order to determine whether intestinal injury was caused by kidney damage in the hyperuricemic mice, the levels of uremic toxins, such as IS and PCS in the serum were measured. The results indicated that their serum levels were markedly increased in the hyperuricemic group compared with the controls. The effects of notably increased uremic toxin levels on intestinal damage may not fully account for the observed intestinal injury in these models.

Numerous studies have demonstrated that the increased production of inflammatory cytokines has a negative impact on intestinal mucosal barrier and epithelial function $(20,38)$. For example, Xin et al (39) showed that impaired intestinal barrier in a rat model of chronic obstructive pulmonary disease was associated with the intestinal inflammatory response, in which elevated levels of the cytokines interferon- $\gamma$, TNF- $\alpha$ and IL- 8 were observed. In vitro experiments have also revealed that TNF- $\alpha$ may result in structural changes to the tight junctions by decreasing occludin and ZO-1 expression (40). In humans, patients with hyperuricemia were found to have higher levels of inflammatory cytokine levels in the serum compared with the healthy controls (41). Similar results were observed in rabbits, in which the addition of exogenous UA to the intestinal lumen promoted an intestinal inflammatory reaction (11). In the present study, elevated production of the inflammatory cytokines TNF- $\alpha$ and IL- 6 in the serum and intestinal homogenates were detected in the hyperuricemic mice. These results suggest there was a positive association between the increased levels of inflammatory cytokines and intestinal epithelial tight junction dysfunction. Therefore, hyperuricemia may affect intestinal barrier function through the initiation of inflammatory responses in the intestine. Further studies are required to determine the precise mechanism underlying intestinal mucosal barrier damage in hyperuricemic mice.

In conclusion, the present study demonstrated the presence of a damaged intestinal barrier and enhanced intestinal permeability in a mouse model of hyperuricemia. The impaired intestinal barrier function may be associated with the inflammatory process induced by UA. The findings of the present study may provide novel insight into organ damage a consequence of hyperuricemia. 


\section{Acknowledgements}

Not applicable.

\section{Funding}

The present study was supported by the National Natural Science Foundation of China (grant no. 81502025), the Natural Science Foundation of Shandong Province (grant no. ZR2018MH004) and China's Post-doctoral Science Fund (grant no. 2018M632631).

\section{Availability of data and materials}

The datasets used and/or analyzed during the current study are available from the corresponding author on reasonable request.

\section{Authors' contributions}

YG and ZT designed the study. YG, HL and YY performed the experiments. HL, ZL, CL, YC and CJ contributed to data analysis. YG and HL drafted the manuscript. YG, YY, and ZT revised the manuscript. All authors have given approval to the final version of the manuscript.

\section{Ethics approval and consent to participate}

The present study was approved by the Animal Research Ethics Committee of the Affiliated Hospital of Qingdao University and the approval number was AHQU20140612A.

\section{Patient consent for publication}

Not applicable.

\section{Competing interests}

The authors declare that they have no competing interests.

\section{References}

1. Mandal AK and Mount DB: The molecular physiology of uric acid homeostasis. Annu Rev Physiol 77: 323-345, 2015.

2. Álvarez-Lario B and Macarrón-Vicente J: Uric acid and evolution. Rheumatology 49: 2010-2015, 2010.

3. Liu R, Han C, Wu D, Xia X, Gu J, Guan H, Shan Z and Teng W: Prevalence of hyperuricemia and gout in mainland China from 2000 to 2014: A systematic review and meta-analysis. Biomed Res Int 2015: 762820, 2015.

4. Trifiro G, Morabito P, Cavagna L, Ferrajolo C, Pecchioli S, Simonetti M, Bianchini E, Medea G, Cricelli C, Caputi AP and Mazzaglia G: Epidemiology of gout and hyperuricaemia in Italy during the years 2005-2009: A nationwide population-based study. Ann Rheum Dis 72: 694-700, 2013.

5. Robinson PC: Gout-an update of aetiology, genetics, co-morbidities and management. Maturitas 118: 67-73, 2018.

6. Johnson RJ, Bakris GL, Borghi C, Chonchol MB, Feldman D, Lanaspa MA, Merriman TR, Moe OW, Mount DB and Sanchez Lozada LG: Hyperuricemia, acute and chronic kidney disease, hypertension, and cardiovascular disease: Report of a scientific workshop organized by the national kidney foundation. Am J Kidney Dis 71: 851-865, 2018.

7. Bonakdaran S and Kharaqani B: Association of serum uric acid and metabolic syndrome in type 2 diabetes. Cur Diabetes Rev 10: 113-117, 2014.
8. Yun Y, Yin H, Gao Z, Li Y, Gao T, Duan J, Yang R, Dong X, Zhang L and Duan W: Intestinal tract is an important organ for lowering serum uric acid in rats. PLoS One 12: e0190194, 2017.

9. Xu X, Li C, Zhou P and Jiang T: Uric acid transporters hiding in the intestine. Pharm Biol 54: 3151-3155, 2016.

10. Fini MA, Elias A, Johnson RJ and Wright RM: Contribution of uric acid to cancer risk, recurrence and mortality. Clin Transl Med 1: 16, 2012.

11. Crane JK and Mongiardo KM: Pro-inflammatory effects of uric acid in the gastrointestinal tract. Immunol Invest 43: 255-266, 2014.

12. Lu J, Hou X, Yuan X, Cui L, Liu Z, Li X, Ma L, Cheng X, Xin Y, Wang C, et al: Knockout of the urate oxidase gene provides a stable mouse model of hyperuricemia associated with metabolic disorders. Kidney Int 93: 69-80, 2018.

13. Lindén SK, Florin TH and McGuckin MA: Mucin dynamics in intestinal bacterial infection. PLoS One 3: e3952, 2008.

14. Bustin SA, Benes V, Garson JA, Hellemans J, Huggett J, Kubista M, Mueller R, Nolan T, Pfaffl MW, Shipley GL, et al: The MIQE guidelines: Minimum information for publication of quantitative real-time PCR experiments. Clin Chem 55: 611-622, 2009.

15. Livak KJ and Schmittgen TD: Analysis of relative gene expression data using real-time quantitative PCR and the 2(-Delta Delta C(T)) method. Methods 25: 402-408, 2001.

16. Dvorak K, Higgins A, Palting J, Cohen M and Brunhoeber P: Immunohistochemistry with Anti-BRAF V600E (VE1) mouse monoclonal antibody is a sensitive method for detection of the BRAF V600E mutation in colon cancer: Evaluation of 120 cases with and without KRAS mutation and literature review. Pathol Oncol Res 25: 349-359, 2019.

17. Wang Y, Yang F, Xue J, Zhou X, Luo L, Ma Q, Chen YF, Zhang J, Zhang SL and Zhao L: Antischistosomiasis liver fibrosis effects of chlorogenic acid through IL-13/miR-21/Smad7 signaling interactions in vivo and in vitro. Antimicrob Agents Chemother 61: pii: e01347-16, 2017.

18. Zhao Y, Qin GX, Sun Z, Che D, Bao N and Zhang X: Effects of soybean agglutinin on intestinal barrier permeability and tight junction protein expression in weaned piglets. Int J Mol Sci 12: 8502-8512, 2011

19. Song $C$ and Zhao X: Uric acid promotes oxidative stress and enhances vascular endothelial cell apoptosis in rats with middle cerebral artery occlusion. Biosci Rep 38: pii: BSR20170939, 2018.

20. Shen L: Tight junctions on the move: Molecular mechanisms for epithelial barrier regulation. Ann NY Acad Sci 1258: 9-18, 2012.

21. Wang H, Zhang Q, Niu Y, Zhang X and Lu R: Surface-layer protein from Lactobacillus acidophilus NCFM attenuates tumor necrosis factor- $\alpha$-induced intestinal barrier dysfunction and inflammation. Int J Biol Macromol 136: 27-34, 2019.

22. Morisaki T: Animal models for abnormal purine metabolism. Nihon Rinsho 61 (Suppl 1): S482-S486, 2003 (In Japanese).

23. Zhu Y, Peng $X$ and Ling G: An update on the animal models in hyperuricaemia research. Clin Exp Rheumatol 35: 860-864, 2007.

24. Sorensen LB: Role of the intestinal tract in the elimination of uric acid. Arthritis Rheum 8: 694-706, 1965.

25. Matsuo H, Tsunoda T, Ooyama K, Sakiyama M, Sogo T, Takada T, Nakashima A, Nakayama A, Kawaguchi M, Higashino T, et al: Hyperuricemia in acute gastroenteritis is caused by decreased urate excretion via ABCG2. Sci Rep 6: 31003, 2016.

26. Ruan Z, Liu S, Zhou Y, Mi S, Liu G, Wu X, Yao K, Assaad H, Deng Z, Hou Y, et al: Chlorogenic acid decreases intestinal permeability and increases expression of intestinal tight junction proteins in weaned rats challenged with LPS. PLoS One 9: e97815, 2014.

27. Xu R, Lei Y, Shi J, Zhou YJ, Chen YW and He ZJ: Effects of lactadherin on plasma D-lactic acid and small intestinal MUC2 and claudin-1 expression levels in rats with rotavirus-induced diarrhea. Exp Ther Med 11: 943-950, 2016.

28. Marchiando AM, Shen L, Graham WV, Edelblum KL, Duckworth CA, Guan Y, Montrose MH, Turner JR and Watson AJ: The epithelial barrier is maintained by in vivo tight junction expansion during pathologic intestinal epithelial shedding. Gastroenterology 140: 1208-1218.e1-e2, 2011.

29. Swidsinski A, Loening-Baucke V, Theissig F, Engelhardt H, Bengmark S, Koch S, Lochs H and Dörffel Y: Comparative study of the intestinal mucus barrier in normal and inflamed colon. Gut 56: 343-350, 2007. 
30. Van Spaendonk H, Ceuleers H, Witters L, Patteet E, Joossens J, Augustyns K, Lambeir AM, De Meester I, De Man JG and De Winter BY: Regulation of intestinal permeability: The role of proteases. World J Gastroenterol 23: 2106-2123, 2017.

31. Odenwald MA and Turner JR: Intestinal permeability defects: Is it time to treat? Clin Gastroenterol Hepatol 11: 1075-1083, 2013.

32. Meng Y, Zhang Y, Liu M, Huang YK, Zhang J, Yao Q, Zhao YL and Xiong JJ: Evaluating intestinal permeability by measuring plasma endotoxin and diamine oxidase in children with acute lymphoblastic leukemia treated with high-dose methotrexate. Anticancer Agents Med Chem 16: 387-392, 2016.

33. Honzawa $Y$, Nakase $H$, Matsuura $M$ and Chiba T: Clinical significance of serum diamine oxidase activity in inflammatory bowel disease: Importance of evaluation of small intestinal permeability. Inflamm Bowel Dis 17: E23-E25, 2011.

34. Lau WL, Kalantar-Zadeh K and Vaziri ND: The Gut as a source of inflammation in chronic kidney disease. Nephron 130: 92-98, 2015.

35. Vaziri ND, Yuan J, Rahimi A, Ni Z, Said H and Subramanian VS: Disintegration of colonic epithelial tight junction in uremia: A likely cause of CKD-associated inflammation. Nephrol Dial Transplant 27: 2686-2693, 2012.

36. Adesso S, Ruocco M, Rapa SF, Piaz FD, Raffaele Di Iorio B, Popolo A, Autore G, Nishijima F, Pinto A and Marzocco S: Effect of indoxyl sulfate on the repair and intactness of intestinal epithelial cells: Role of reactive oxygen Species' release. Int J Mol Sci 20: pii: E2280, 2019.
37. Vaziri ND, Zhao YY and Pahl MV: Altered intestinal microbial flora and impaired epithelial barrier structure and function in CKD: The nature, mechanisms, consequences and potential treatment. Nephrol Dial Transplant 31: 737-746, 2016.

38. Luissint AC, Parkos CA and Nusrat A: Inflammation and the intestinal barrier: Leukocyte-epithelial cell interactions, cell junction remodeling, and mucosal repair. Gastroenterology 151: 616-632, 2016.

39. Xin X, Dai W, Wu J, Fang L, Zhao M, Zhang P and Chen M: Mechanism of intestinal mucosal barrier dysfunction in a rat model of chronic obstructive pulmonary disease: An observational study. Exp Ther Med 12: 1331-1336, 2016.

40. Wang F, Graham WV, Wang Y, Witkowski ED, Schwarz BT and Turner JR: Interferon-gamma and tumor necrosis factor-alpha synergize to induce intestinal epithelial barrier dysfunction by up-regulating myosin light chain kinase expression. Am J Pathol 166: 409-419, 2005.

41. Zhou Y, Xu GQ, Pu ZY, et al: Relationship between oxidative stress and inflammatory response in young patients with primary hyperuricemia. China J Modern Med 97: e13108, 2017.

This work is licensed under a Creative Commons

Attribution-NonCommercial-NoDerivatives 4.0 International (CC BY-NC-ND 4.0) License. 